\title{
INCIDENCIA DE LA EVALUACIÓN Y LAS METODOLOGíAS DOCENTES EN EL DESARROLLO DE COMPETENCIAS GENÉRICAS
}

\author{
Alicia J Inciarte \\ Universidad de la Costa (Colombia) \\ ainciart1@cuc.edu.co \\ Jesús N García \\ Universidad de León / Universidad de la Costa \\ Edgardo R Sánchez \\ Universidad de la Costa (Colombia) \\ Marcial E Conde \\ Universidad de la Costa (Colombia) \\ Freddy V Marín \\ Universidad de la Costa (Colombia) \\ Judit S Castillo \\ Universidad de la Costa (Colombia) \\ Judit García-Martín \\ Universidad de Salamanca
}

Fecha de Recepción: 23 Enero 2019

Fecha de Admisión: 30 Abril 2019

\section{RESUMEN}

Este artículo presenta un programa de investigación que lleva a estudiar la incidencia de la evaluación y las metodologías docentes activas usadas y esperadas en la formación universitaria, para el desarrollo de las competencias genéricas comunicativas en los estudiantes de diferentes carreras. En sentido más general, se busca Identificar el uso percibido y esperado, por profesores y estudiantes, de la evaluación y metodologías activas; recopilar estrategias transversales exitosas; describir y comparar los patrones psicoeducativos y su papel en los resultados de aprendizaje y competencias genéricas comunicativas de lectura crítica y construcción textual, a partir de lo cual de llegaría a configurar formación en valoración integral de la enseñanza y aprendizaje que habría de proporcionarse a los docentes de la universidad para que éstos se conviertan en mediadores y promotores de competencias genéricas comunicativas de lectura crítica y construcción textual a través de las asignaturas -across disciplines- en los estudiantes. Se realiza en la Universidad de la Costa en Colombia y se aborda desde dos focos de estudio: detección de necesidades e implementación de estrategias transversales. Se trabajan patrones psicoeducativos (autoeficacia, atribuciones, motiva- 
ción, inteligencia emocional...); también la revisión sistemática de prácticas exitosas internacionales. Los resultados se orientan a la implementación de estrategias para la innovación curricular de las asignaturas y mejora docente y de los resultados académicos y de aprendizaje; así mismo, para el aumento de conocimientos teóricos y tecnológicos instruccionales pedagógicos y didácticos como base para la toma de decisiones en la segunda fase de validación y contrastación de estrategias transversales. "Proyecto de Investigación Competitivo CONV-ÍNDEX Núm. 13-2018, Código Proyecto INV. 150-01-007-13 Universidad de la Costa (Colombia) - Universidad de León (España). Evaluado por la ACAC (MEN Colombia)"

Palabras claves: evaluación; metodologías activas; transversalización asignaturas; lectura crítica; construcción textual

\begin{abstract}
Impact of the evaluation and teaching methodologies in the development of generic competences. This paper presents a research program that leads to study the impact of evaluation and active teaching methodologies used and expected in university education, for the development of generic communicative skills in students of different careers. In a more general sense, it seeks to identify the perceived and expected use, by teachers and students, of the evaluation and active methodologies; collect successful cross-cutting strategies; describe and compare psychoeducational patterns and their role in the learning outcomes and generic communicative competences of critical reading and textual construction, from which would come to configure training in comprehensive assessment of teaching and learning to be provided to teachers of the university so that they become mediators and promoters of generic communicative skills of critical reading and textual construction through the subjects -across disciplines- in the students. It is carried out at the Universidad de la Costa in Colombia and it is approached from two foci of study: detection of needs and implementation of transversal strategies. Psychoeducational patterns are used (self-efficacy, attributions, motivation, emotional intelligence ...); also the systematic review of international successful practices. The results are oriented to the implementation of strategies for the curricular innovation of the subjects and teacher improvement and of the academic and learning results; likewise, for the increase of pedagogical and didactic instructional and technological knowledge as a basis for decision making in the second phase of validation and testing of transversal strategies. "Competitive Research Project CONV-ÍNDEX No. 13-2018, Project Code INV. 150-01-007-13 University of the Coast (Colombia) - University of León (Spain). Evaluated by the ACAC (MEN Colombia) “
\end{abstract}

Keywords: evaluation; active methodologies; across disciplines; critical reading; textual construction

\title{
INTRODUCCIÓN
}

El estudio de la valoración integral de los procesos de enseñanza y aprendizaje se realiza mediante sistemas de evaluación diversos y comprehensivos, que permitan la detección de situación y necesidades; siendo dicha información la base para la toma de decisiones didácticas e instruccionales en la práctica cotidiana en las disciplinas, en una encrucijada compleja de hacia dónde va la evaluación (Tamayo, Niño, Cardozo \& Bejarano, 2018), de la educación superior en Colombia (OCDE, 2016), considerando las limitaciones de la valoración de las competencias (Barnet, 2001; Díaz-Flórez, 2018) siendo más importante la calidad de la enseñanza que los propios sistemas de evaluación (Ravitch, 2013); especialmente relevante para la formación inicial del profesorado en Colombia (Arias-Gómez, Díaz, Garzón, León, Rodríguez \& Valbuena, 2018); es decir, preservice teachers (García-Martín \& García, 2017). La cuestión clave que persiste en este debate es la de obte- 
ner evidencias empíricas validadas, más allá de las propias reflexiones que pueden guiar el proceso de investigación.

Se trabajan medidas perceptivas por profesores y estudiantes de variables sobre SSEV y MMAC utilizadas por los profesores, y de las estrategias directamente potenciadoras de las competencias genéricas (CCGG), además de sugerencias que les gustaría estuvieran (esperado) vs., a efectivamente realizado (trabajado). Se vaolra la introducción de medidas de variables personales y patrones psicoeducativos como autoeficacia (AE) hacia la práctica de profesores ante SSEV-MMAC-instrucción de CCGG, motivación (Mot), estrategias de afrontamiento ante problemas (EAP), HHSS, inteligencia emocional (IE), atribuciones de causa (Atr), desempeño social -DS-, o medidas de uso de herramientas de la web 2.0-4.0 (HEWE) (García M \& García, 2013; 2015). Además, habría que estudiar la viabilidad de incluir otras medidas relativas a estrategias de aprendizaje y motivacion (CEAM), estilos de aprendizaje (CEPEA) o incluso de personalidad (FF). Junto con la obtención sistemática de datos sobre resultados académicos de los alumnos de los profesores estudiados (niveles de logro) para ver si hay relación con los resultados académicos (y otras variables psicoeducativas y medidas anteriores). Se están implementamdo online con la aplicación de encuestación profesional SurveyMonkey.

Se presenta un programa de investigación a cuatro años, de identificación de sistemas de evaluación y metodologías docentes activas, usadas y esperadas, así como de estrategias exitosas de fomento de competencias genéricas lectura crítica y construcción textual a través de asignaturas. El marco teórico parte de cinco ejes integrados, enseñanza y aprendizaje centrado en competencias considerando el debate sobre sus límites; perspectiva instruccional psicológica-educativa -pedagógica y didáctica-; metodologías docentes activas dentro de una web ubicua (web 4.0); tecnología de validación de instrumentos; controles de calidad - indicadores IBE-. Para ello se plantean dos tipos de estudios, de detección y necesidades y de implementación de estrategias transversales, y que se van solapando en su desarrollo para lograr las metas propuestas. Se presenta el plan de los estudios de detección de necesidades (2019-2020).

En la primera fase se han implementado estudios descriptivos de detección de situación y necesidades sobre los SSEV y MMAC deseadas y desarrolladas y comparativos en torno a variables de agrupamiento nucleares; junto con estudios de validación de instrumentos; y comparativos con variables personales y patrones psicoeducativos (autoeficacia, atribuciones, motivación, inteligencia emocional...); y estudios de revisión sistemática de prácticas exitosas internacionales aplicables en la segunda fase. Se esperan obtener datos relevantes para la innovación curricular de las asignaturas y mejora docente y de los resultados académicos y de aprendizaje; y en el aumento de conocimientos teóricos y tecnológicos instruccionales pedagógicos y didácticos como base para la toma de decisiones en la segunda fase de validación y contrastación de estrategias transversales.

Se parte de la pregunta de investigación: ¿cuál sería la formación en valoración integral de la enseñanza y aprendizaje que habría de proporcionarse a los docentes de la universidad para que éstos se conviertan en mediadores y promotores de competencias genéricas comunicativas de lectura crítica y construcción textual a través de las asignaturas -across disciplines- en los estudiantes?

Para ello, tras revisar los antecedentes exitosos implementados en la CUC (CUC-AIA, 2018), relativos (i) al factor 2 de los estudiantes (completado en el factor 9 de bienestar institucional), ilustrado con las estrategias sobre permanencia y deserción estudiantil a través del Programa de Acompañamiento, Seguimiento de la Permanencia Estudiantil (PASPE) y estudio de los factores determinantes de la deserción temprana (Fragozo, 2009). y tardías (Fragozo, 2013), interesando sobre todo considerar las estrategias de aprender a aprender, refuerza-T y bienestar en la clase; (ii) 
los del factor 3 de profesorado, interesando las estrategias de la buena práctica docente, la capacitación docente, la docencia inicial y el plan de acompañamiento docente (PAD); (iii) y las relativas al factor 6 de autoevaluación y autorregulación, siendo relevante el programa "Saber te lleva lejos" del Centro de Excelencia Docente, y las estrategias de formación y acompañamiento y la elaboración de pruebas diagnósticas.

Además, los antecedentes empíricos internacionales, de doble naturaleza, (i) los procedentes de los indicadores de programas validados empíricamente como las mejores prácticas educativas, tanto para competencias genéricas específicas como para la mejora de calidad de docentes y directivos; y (ii) todo el desarrollo internacional relativo a la implementación de competencias genéricas como la comunicativa u otras a través de las disciplinas (ej., literacy across the disciplines, Scott, McTigue, Miller \& Washburn, 2018; writing as a learning activity, Klein \& Boscolo, 2016).

La justificación de la necesidad de estas investigaciones se viene evidenciando en varios argumentos de calado. Por una parte, la constatación de que los estudiantes de la CUC que realizan las pruebas Saber Pro puntúan más bajo de lo deseable (CUC-AIA, 2018; CUC-Departamento de Estadística, 2017), si bien explicable, en parte, por la procedencia del alumnado de la CUC mayoritario de estratos 1-3, lo que el factor status socioeconómico debe de ser considerado en la valoración de este dato, frente a la procedencia del alumnado de otras universidades (estratos 4-6) (CUCAIA, 2018; CUC-Departamento de Estadística, 2017). Ello ha llevado a la implementación de estrategias diversas con foco en la mejora de los sistemas de evaluación de todas las asignaturas de la universidad, el uso de rúbricas, el acompañamiento de actividades, y otras recogidas en el último autoinforme de acreditación (CUC-AIA, 2018), incluyendo la reciente puesta en marcha de la estrategia "10\% más" en todas las asignaturas de todos los estudios y que incluye este porcentaje de calificación en las competencias genéricas relacionadas con la asignatura en cuestión; o las experiencias fortalecidas recientemente sobre la transversalización de las humanidades. Si bien, la mayoría de las medidas son apreciadas positivamente por los grupos de interés implicados, falta una validación formal de su eficacia y eficiencia, más allá de una constatación impresionística.

Para responder a la pregunta, se prevé en una primera fase hacer un análisis de situación sobre el uso de sistemas de evaluación (SSEV) y metodologías activas (MMAC) efectivamente utilizados por los docentes y de las necesidades formativas requeridas. $Y$ en una segunda fase se trataría de diseñar e implementar estrategias directamente transversales sobre los alumnos en las diferentes materias y evaluar su eficacia. Parece pertinente la realización de estudios descriptivos sobre la situación y necesidades formativas; así como estudios instruccionales y didácticos en torno a la implementación de estrategias transversales que incidan no solo en la valoración del proceso de enseñanza y aprendizaje, sino también en las metodologías docentes activas, de forma general y específica para los tipos de asignaturas. Es decir, el foco se centraría en las competencias genéricas comunicativas de LC y de CT ilustrado por focos de literacy across the disciplines (Scott et al., 2018), writing as a learning activity (Klein \& Boscolo, 2016), 0 autorregulación de los aprendizajes y rendimiento académico (Li, Ye, Tang, Zhou \& Hu, 2018).

El objetivo general en que se concreta la respuesta a la pregunta de investigación consisten en identificar el uso percibido y esperado, por profesores y estudiantes, de la evaluación y metodologías activas; recopilar estrategias transversales exitosas; y describir y comparar los patrones psicoeducativos y su papel en los resultados de aprendizaje y competencias genéricas comunicativas de lectura crítica y construcción textual. Y se materializa en los siguientes objetivos específicos: (i) Detección de situación de SSEV y MMAC utilizados y necesidades formativas, desde la perspectiva de los profesores y de los estudiantes. E identificar los SSEV y MMAC que esperan/desean implementar vs., las efectivamente aplicadas. (ii) Analizar variables psicoeducativas relativas a autoefica- 
cia percibida de los profesores en los SSEV y MMAC e instrucción en LC y CT, y otras posibles como motivación y estrategias de aprendizaje, 0 afrontamiento, estilos de aprendizaje, atribuciones de causa, inteligencia emocional y competencia social, u otras variables de personalidad. (iii) Analizar el uso de herramientas de la web 2.0-4.0 y competencia digital con fines de eLearning y otros, en relación con los SSEV y MMAC y/o con variables y patrones psicoeducativos, por profesores y estudiantes. (iv) Analizar estudios internacionales que contrastan estrategias pedagógicas-didácticasinstruccionales que han funcionado en otros contextos dentro de actividades transversales significativas en las asignaturas - literacy across disciplines-content, con foco en self-regulated learning - referidas a las competencias genéricas LC y CT. (v) En una segunda fase del programa de investigación (2020-2021-2022) diseñar, adaptar y validar actuaciones y estrategias transversales sobre los estudiantes a través de las materias-contenido y evaluar su eficacia y papel mediador y modulador de las variables o patrones psicoeducativos.

Este programa de investigación se viene realizando en la Universidad de la Costa en Colombia y se aborda desde dos focos de estudio: detección de necesidades e implementación de estrategias transversales. Se trabajan patrones psicoeducativos (autoeficacia, atribuciones, motivación, inteligencia emocional...); también la revisión sistemática de prácticas exitosas internacionales.

\section{MÉTODO}

\section{Participantes}

\section{Profesores}

Hasta ahora han participado en la primera fase del programa 249 profesores de la Universidad de la Costa (Colombia) de los cuales 182 ( $N=182,113$ hombres y 69 mujeres), aceptaron participar voluntariamente respondiendo a la totalidad del cuestionario ad hoc, EMICOG en su versión docente, enviado on line. En edades comprendidas entre 24 y 73 años. Su experiencia laboral es variable y relacionada a la categoría profesional (catedrático, asistente, adjunto y titular). Parte de estos profesores tiene experiencia laboral externa al sector académico, el 30,5\% desempeñan un ejercicio profesional libre y externo, el 12,8\% trabajan en una empresa externa y el 9,9\% ejercen como docentes de enseñanza no universitaria (García-Martín, García, Inciarte, Marín, Sánchez \& Conde, 2019a).

\section{Estudiantes}

En total, 3647 estudiantes ( $N=1336$ hombres y 1439 mujeres) respondieron voluntariamente el cuestionario EMICOG - versión estudiantes, on line, con edades comprendidas entre los 16 y 62 años. Cursan las carreras de Arquitectura, Ciencias Económicas, Ciencias Humanas y Sociales, Derecho e Ingeniería. El 13,5 \% de los estudiantes participantes [ $\mathrm{N}=375$ (210 hombres y 165 mujeres)] indican trabajar a tiempo parcial, el $20,4 \%$ [N = 566 (287 hombres y 279 mujeres)] a tiempo completo y el $66,1 \%$ no trabajaba [ $\mathrm{N}=1834$ (839 hombres y 995 mujeres)]. Con respecto a su estratficación económica el 26,4\% de los encuestados [ $\mathrm{N}=732$ (339 hombres y 393 mujeres)] manifiestan pertenecer al primer estrato denominado bajo-bajo; el $36 \%$ [N = 999 (480 hombres y 519 mujeres)] al segundo o bajo, 28,3\% [N = 785 (381 hombres y 401 mujeres)] al tercero 0 medio-bajo, el 7,4\% [ $\mathrm{N}=205$ (108 hombres y 97 mujeres)] al cuarto o medio, el 1,4 \% [ $\mathrm{N}=40$ (19 hombres y 21 mujeres)] al quinto 0 alto y el $0,5 \%$ [ $N=14$ (6 hombres y 8 mujeres)] restante al sexto 0 alto-alto, están cursando los últimos cinco semestres de la carrera (García-Martín, García, Inciarte, Marín, Sánchez \& Conde, 2019b).

\section{Procedimiento}

Tras una revisión de los instrumentos disponibles a nivel internacional, se seleccionaron los ins- 
trumentos y variables a medir. Para los profesores y estudiantes se han evaluado en esta primera fase, los datos demográficos y generales para utilizar como variables de agrupamiento y contraste; el grado de conocimiento e interés en variables claves investigadas (modelo de competencias, transversalización de la lectura crítica y construcción textual; las variables relacionadas con las técnicas y estrategias implementadas habitualmente en las diferentes asignaturas tanto para la lectura crítica como para la construcción textual en sus diferentes facetas de procesos, géneros textuales, medios. Además, otro foco de evaluación fue la evaluación y enseñanza utilizada de forma habitual en las asignaturas en relación con las competencias genéricas de lectura crítica y construcción textual. También, se analiza el patrón de variables psicoeducativas de autoeficacia, inteligencia emocional, motivación y atribuciones, la dimensión social y las estrategias de afrontamiento habituales ante problemas. Y por último, las necesidades formativas identificadas en relación con la enseñanza y aprendizaje y con las herramientas de la web (GarcíaM et al., 2019a). De forma adicional, para los estudiantes se incluyó el Cuestionario de Evaluación de Procesos de Estudio y de Aprendizaje (CEPEA) para medir los estilos de aprendizaje; las actitudes hacia la lectura crítica y la construcción textual, y la ansiedad ante la lectura crítica y la construcción textual (ante la evaluación, ante la temporalidad, ante la comprensión de las tareas, ante situaciones de la vida real y general) (GarcíaM et al., 2019b).

Cabe la opción de incluir medidas de grupos focales, entrevistas abiertas y registros observacionales, codificados en SSCCE\&ME (segunda fase).

\section{Análisis de datos}

Se están implementando tres tipos de análisis con SPSSv25 y AMOS (ULE), y/0 con paquetes R+ 0 MPlus u otros adecuados. Un primer tipo de análisis con el SPSS, son de tipo descriptivo de constatación de normalidad de variables, de análisis de tablas y diferencias significativas, de posible transformación de variables y escalas, de análisis paramétricos y no paramétricos. Un segundo tipo de análisis de tipo multivariado, con el SPSS, de tipo comparativo en función de variables de agrupamiento relevantes (curso, edad, niveles de logro, áreas y materias, patrones psicoeducativos diferenciales), implementando modelos lineales generales, análisis funcionales discriminantes, análisis de conglomerados, análisis de regresión y predictivos. En tercer lugar, análisis de validación de instrumentos, con el SPSS y AMOS, relativo a análisis de ítems, análisis factorial exploratorio (AFE) y análisis factorial confirmatorio (AFC), para la valoración de la fiabilidad (alfas, omegas McDonald / fiabilidad compuesta, varianza media extractada), validez de constructo, estructural, discriminante, predictiva. Y por último, habría que estudiar la viabilidad de implementar sistemas de ecuaciones estructurales (SEM) para determinar el papel de variables mediadoras y/o moduladoras que permitan avanzar en modelos explicativos y que sirvan de base para el establecimiento en la segunda fase de énfasis instruccionales en las variables relevantes (AMOS, MPlus).

\section{DISCUSIÓN Y CONCLUSIONES}

Este programa de investigación en proceso, sobre la Incidencia de la Evaluación y las metodologías docentes en el desarrollo de competencias genéricas, se realiza desde la percepción de los profesores y estudiantes, a través de cuestionarios on line. Los resultados apuntan a que de la indagación con los profesores se llegará a determinar las metodologías docentes utilizadas con mayor frecuencia e identificar las posibles razones o motivos de dicha preferencia. También se busca definir necesidades formativas del profesorado, para ser propuestas a la institución y contribuir con mejorar le enseñanza y desarrollo de las competencias genéricas.

Por su parte la consulta a estudiantes permitirá identificar y analizar sus percepciones y creen- 
cias acerca de las distintas metodologías docentes universitarias, de acuerdo a las asignaturas que han cursado, así mismo valorar la actuación institucional en la mejora de los indicadores académicos.

Los resultados permitirán fundamentar la implementación de estrategias para la innovación curricular de las asignaturas y mejora docente y de los resultados académicos y de aprendizaje; así mismo, para el aumento de conocimientos teóricos y tecnológicos instruccionales pedagógicos y didácticos como base para la toma de decisiones en la segunda fase de validación y contrastación de estrategias transversales (Miller, Scott y McTigue, 2016; Peng, Wang, Wang y Lin, 2019).

Respecto al impacto y efectos esperados, son de diferente naturaleza. Por una parte, a nivel científico se están obteniendo nuevos instrumentos de evaluación validados utilizables por otros investigadores y para la evaluación de la implementación de innovaciones docentes y curriculares. Además, los estudios descriptivos y comparativos están aportando datos valiosos sobre la situación del uso de SSEV y MMAC y su relación con variables personales e instruccionales del tipo de patrones psicoeducativos y perfiles de características asociados a variables de agrupamiento claves como niveles de logro, áreas de procedencia, que servirán para la toma de decisiones basadas en la evidencia científica, indicando qué SSEV y MMAC y o perfiles son los que mejor predicen mayor rendimiento en los alumnos, o mayor satisfacción. Además, para la siguiente fase de implementación, validación y contrastación de estrategias transversales de fomento, se identificarán las basadas en la evidencia empírica y su relación con perfiles y características personales y más recomendables, a la vez que aumentarán los conocimientos teóricos y su desarrollo tecnológico sobre la naturaleza y complejidad del problema, sirviendo de fundamento para la implementación de innovaciones curriculares, docentes e instruccionales. Se asume la consideración de las reflexiones y debates internacionales y en Colombia, sobre la evaluación (Tamayo et al., 2018), los límites de las competencias (Barnet, 2001; Díaz-Flórez, 2018), en el contexto de la situación de la formación inicial de los profesores y la educación superior en Colombia (Arias-Gómez et al., 2018; GarcíaM \& García, 2017; OCDE, 2016), priorizando la calidad de la enseñanza y aprendizaje como guía para el estudio presente (Ravitch, 2013). Por otra parte, el impacto en diversos contextos sociales, en relación con la mejora y diversificación de los SSEV y MMAC, percibidas, realizadas y deseadas o esperadas, por profesores y estudiantes, la identificación de las necesidades formativas y, en la siguiente fase de implementación de estrategias transversales de fomento de CCGG, incidirá en la mejora de la calidad docente, mejores RRAA y mayor satisfacción en los estudiantes, y por lo tanto mejor calidad de vida y una respuesta en la sociedad con profesionales más competentes y respuestas de mayor calidad en la prestación de servicios (Widlund, Tuominen, y Korhonen, 2018). Además, desde la perspectiva del medio ambiente 0 ambiental el programa de investigación incidirá en una mayor conciencia medioambiental, producto de la formación integral con el fomento de estrategias transversales de mejora de las CCGG, incidiendo en una posición más responsable y crítica con el medio ambiente. Otro impacto añadido tiene que ver con la incidencia en la productividad y en la competitividad, pues se espera mejorar y diversificar los SSEV y MMAC desarrollados y compartidos por docentes y estudiantes; una mayor satisfacción y mejores resultados académicos y en CCGG de Ios estudiantes con un efecto positivo en los resultados (USOE, 2019; Uttl, White, y Wong., 2017; van Ockenburg, van Weijen y Rijlaarsdam, 2019). Y en la segunda fase, con la validación de estrategias transversales exitosas, se potenciarán los resultados esperables anteriores. Se espera un impacto de investigación con aumento de conocimiento científico y tecnológico; un impacto en la docencia con innovación docente y resultados académicos; e impacto en acciones de extensión para la comunidad educativa en general; (Widlund et al., 2018; Xie, Wang y Hu, 2018; Zeng, Chen, Cheung y Peng, 2019). 


\section{INCIDENCIA DE LA EVALUACIÓN Y LAS METODOLOGíAS DOCENTES EN EL DESARROLLO DE COMPETENCIAS GENÉRICAS}

\section{REFERENCIAS BIBLIOGRÁFICAS}

Arias-Gómez, D. H., Díaz-Flórez, O. C., Garzón-Barragán, I., León-Palencia, A. C., Rodríguez-Ávila, S. P., Valbuena-Ussa, E. 0. (2018) Entre las exigencias de calidad y las condiciones de desigualdad: Formación inicial de profesores en Colombia. Bogotá: UPN

Barnet, R (2001). Los límites de la competencia. El conocimiento, la educación superior y la sociedad. Barcelona: Gedisa

CUC (2018). Autoinforme de Acreditación. Barranquilla: CUC

CUC-Departamento de Estadística (2017a). Informe de Resultados de las Pruebas en SaberPro de Ios estudiantes de la CUC. Barranquilla: CUC

CUC-Departamento de Estadística (2017b). Informe laboral de los estudiantes de la

Díaz-Flórez, 0. C. (2018). Las competencias en la educación superior. Debates contemporáneos. Tesis Doctoral. Bogotá: UPN

Fragozo-Torres, Z. (2009). Estudio de causas e indicadores de la Deserción académica de los estudiantes, cohorte 2006 II - Periodos comprendidos entre 2007 II al 2012 II. Barranquilla: Universidad de la Costa.

Fragozo, Z. (2011) Causas e Indicadores de la Deserción Académica de los Estudiantes de la Corporación Universitaria de la Costa CUC Cohortes 2006 - II a 2011 - 1. Avance de Investigación. GIPIDES. Barranquilla: Universidad de la Costa

García-Martín, J., y García-Sánchez, J. N. (2013). Patterns of web 2.0 tool use among young Spanish people. Computers and Education, 67(3), 105-120. doi: 10.1016/j.compedu.2013.03.003

García-Martín, J., y García-Sánchez, J. N. (2015). Use of Facebook, Tuenti, Twitter and Myspace among young Spanish people. Behaviour \& Information Technology, 34(7), 685-703. doi: 10.1080/0144929X.2014.993428

García-Martín, J., y García-Sánchez, J. N. (2017). Pre-service teachers' perceptions of the competence dimensions of digital literacy and of psychological and educational measures. Computers \& Education, 107, 54-67. doi: 10.1016/j.compedu.2016.12.010

García-Martín, J., y García-Sánchez, J. N. (2018). The instructional effectiveness of two virtual approaches: processes and product. Journal of Psychodidactics, 23(2), 117-127. doi: 10.1016/j.psicod.2018.02.002

García-Martín, J., García, J. N., Inciarte, A. J., Marín, F. V., Sánchez, E. R. y Conde, M. E. (2019a). Sistemas de evaluación y etodologías docentes y su incidencia en las competencias genéricas (EMICOG - profesores). International Journal of Developmental and Educational Psychology, 31 (1)

García-Martín, J., García, J. N., Inciarte, A. J., Marín, F. V., Sánchez, E. R. y Conde, M. E. (2019b). Sistemas de evaluación y etodologías docentes y su incidencia en las competencias genéricas (EMICOG - estudiantes). International Journal of Developmental and Educational Psychology, $31(1)$

Klein, P. D., \& Boscolo, P. (2016). Trends in Research on Writing as a Learning Activity. Journal of Writing Research, 7(3), 311-350. http://dx.doi.org/10.17239/jowr-2016.07.03.01

Li, J., Ye, H., Tang, Y., Zhou, Z., \& Hu, X. (2018). What are the effects of self-regulation phases and strategies for Chinese students? A meta-analysis of two decades research of the association between self-regulation and academic performance. Frontiers in Psychology, 9, 1-13. doi: 10.3389/fpsyg.2018.02434

Miller, D. M., Scott, C. E., \& McTigue, E. M. (2016). Writing in the secondary-level disciplines: A systematic review of context, cognition and content. Educational Psychology Review, doi: 10.1007/s10648-016-9393-z 
OCDE. (2016). Revisión de políticas nacionales de educación La educación en Colombia. Bogotá: Ministerio de Educación Nacional. https://www.mineducacion.gov.co/1759/articles356787_recurso_1.pdf

Peng, P., Wang, T., Wang, C., \& Lin, X. (2019). A Meta-analysis on the Relation between Fluid Intelligence and Reading/Mathematics: Effects of Tasks, Age, and Social Economics Status. Psychological Bulletin, 145(2),189-236. doi: 10.1037/bul0000182

Ravitch, D. (2013). Reign of Error: The Hoax of the Privatization Movement and the Danger to America's Public Schools. New York: Alfred A. Knopf.

Scott, C. E.; McTigue, E. M.; Miller, D. M. \& Washburn, E. K. (2018). The what, when, and how of preservice teachers and literacy across the disciplines: A systematic literature review of nearly 50 years of research. Teaching and Teacher Education 73, 1-13. https://doi.org/10.1016/j.tate.2018.03.010

Tamayo-Valencia, L. A., Niño-Zafra, L. S., Cardozo-Espitia, L. S., \& Bejarano-Bejarano, 0. (2018) ¿Hacia dónde va la evaluación? Aportes conceptuales para pensar y transformar las prácticas de evaluación. Bogotá: IDEP

USOE (2019). OSEP Ideas that Work. Office of Special Education Programs, U.S. Department of Education Recuperado de https://www.osepideasthatwork.org/

Uttl, B., White, C. A., \& Wong Glez, D. (2017): Meta-analysis of faculty s teaching effectiveness: Student evaluation of teaching ratings and student learning are not related. Studies in Educational Evaluation, 54, 22-42. doi: 10.1016/j.stueduc.2016.08.007

van Ockenburg, L., Daphne van Weijen, D., \& Gert Rijlaarsdam, G. (2019). Learning to Write Synthesis Texts: A Review of Intervention Studies. Journal of Writing Research, 401-428 doi: 10.17239/jowr-2019.10.03.01

Widlund, A, Tuominen, H. \& Korhonen, J. (2018). Academic Well-Being, Mathematics Performance, and Educational Aspirations in Lower Secondary Education: Changes Within a School Year. Front. Psychol. 9:297. doi: 10.3389/fpsyg.2018.00297

Xie, C., Wang, M., \& Hu, H. (2018). Effects of Constructivist and Transmission Instructional Models on Mathematics Achievement in Mainland China: A Meta-Analysis. Front. Psychol. 9:1923. doi: 10.3389/fpsyg.2018.01923

Zeng, G., Chen, X., Cheung, H. Y., \& Peng, K. (2019) Teachers' Growth Mindset and Work Engagement in the Chinese Educational Context: Well-Being and Perseverance of Effort as Mediators. Front. Psychol. 10:839. doi: 10.3389/fpsyg.2019.00839 
INCIDENCIA DE LA EVALUACIÓN Y LAS METODOLOGíAS DOCENTES EN EL DESARROLLO DE COMPETENCIAS

GENÉRICAS 